\title{
Gut Microbiota, Combined with Metabolomics, Reveals the Mechanism of Curcumol on Liver Fibrosis in Mice
}

\section{Yang Zheng}

Guangxi University of Chinese Medicine

Jiahui Wang

Guangxi University of Chinese Medicine

Jiaru Wang

Guangdong Medical University

Ruizhu Jiang

Guangxi University of Chinese Medicine

Tianjian Liang

Guangxi University of Chinese Medicine

Tiejian Zhao (D 570041687@qq.com )

Guangxi University of Chinese Medicine

\section{Research Article}

Keywords: Curcumol, Liver fibrosis, Gut microbiota, Metabolomics, Shigella

Posted Date: January 3rd, 2022

DOI: https://doi.org/10.21203/rs.3.rs-1107606/v1

License: () (1) This work is licensed under a Creative Commons Attribution 4.0 International License.

Read Full License 


\section{Abstract}

Background:Liver fibrosis is a reversible pathological process, and its prevention and treatment are of great significance to patients with chronic liver disease. This study combined 16S rRNA analysis of gut microbiota and plasma metabolomics to explore the mechanism of curcumol's effect on liver fibrosis in mice. The results will help to clarify the relationship between the gut microbiota and metabolites in the process of liver fibrosis.

Results:Molecular biological testing found that curcumol could significantly improve the pathological changes of liver tissue and inhibit the occurrence of liver inflammation. Intestinal flora testing found that curcumol could significantly change the abundances of Veillonellaceae, Prerotella_oulorum, and Alistipes_finegoldii. Metabolomics analysis found that curcumol's anti-hepatic fibrosis effect may be related to its regulation of arachidonic acid metabolism. Correlation analysis suggested that curcumol regulated the abundances of Bacteroidota and Bacteroides and participated in the metabolism of Prostaglandin B2.

Conclusions:When liver fibrosis occurs, the intestinal flora and metabolic network will be altered. The effect of curcumol on liver fibrosis may be related to its regulation of intestinal flora and the resulting interference with metabolic pathways, thereby regulating liver inflammation.

\section{Background}

Liver fibrosis is a pathological process common to chronic liver disease that results from multiple factors acting to repair liver damage [1]. As liver damage continues, fibrosis may progress to the irreversible endstage consequence of cirrhosis, the leading cause of morbidity and mortality associated with liver disease worldwide [2]. Nevertheless, fibrosis can be reversed due to the liver's ability to regenerate [3]. Therefore, the early detection and prevention of liver fibrosis is of great clinical importance.

Recent studies have shown that the gut microbiota is involved in the whole range of human physiological processes, including regulation of metabolism and immunity. Obesity, inflammatory bowel disease, diabetes, metabolic syndrome, atherosclerosis, alcoholic liver disease, non-alcoholic fatty liver disease, cirrhosis and liver cancer, and other chronic diseases are all thought to be associated with the gut microbiota [4]. The intestine and liver are closely related in anatomical and biological functions, forming the "gut-liver axis"[5]. Relevant studies have shown that the gut microbiota participates in the formation of liver fibrosis by regulating Toll-like receptor (TLR)-related pathways [6]. Lipopolysaccharide (LPS) is the main component in the cell walls of Gram-negative bacteria. There are a large number of Gram-negative bacteria in the gut microbiota, and LPS can stimulate the liver through the gut-liver axis. LPS is the ligand of TLR4 and functions by activating TLR4 signal transduction, activating NF-KB, promoting the expression

of IL-8, enhancing the inflammatory response, eventually leading to the occurrence of liver fibrosis [7-8]. 
Metabolomics is an emerging field that was developed subsequent to genomics and proteomics. Metabolomics studies the types, quantities, and changes of endogenous metabolites in organisms [9].Metabolomics has been widely used in the diagnosis and monitoring of diseases, providing a new perspective for understanding the pathogenesis of diseases [10-11]. Many people are currently using this technology to find potential disease biomarkers [12]. At present, some metabolomics studies have reported the changes of metabolites in a variety of liver diseases, including hepatocellular carcinoma, non-alcoholic fatty liver, and liver fibrosis [13-15]. Studies have found that lysophosphatidylcholine can be used as a biomarker of liver fibrosis [16].

In recent years, traditional Chinese medicine has achieved remarkable results in the prevention and treatment of liver fibrosis [17]. Curcuma (Curcuma kwangsiensis S.G. Leet and C. F. Liang) is the rhizome of the ginger plant Curcuma or Turmeric. It is used as a medicinal plant material in Guangxi, China. It is called "Ginghgvun" by the local folk and is included in the 2020 edition of the "Chinese Pharmacopoeia." It was first recorded in "The Theory of Medical Properties." In traditional Chinese medicine it is employed in promoting qi and blood, eliminating accumulation of toxins, and relieving pain. It has been used for the treatment of chronic liver disease for many years [18]. It effects include anti-tumor activity, lowering of enzyme activities, and increasing the resistance to liver fibrosis. According to reports, curcumol is the main active component of curcuma [19]. Our previous studies have found that curcumol can inhibit the activity of the TLR4 signaling pathway and thus exhibit anti-liver fibrosis effects [20]. However, the regulatory effect of curcumol on the intestinal flora and metabolites during liver fibrosis is still unclear. This study thus combined the results of gut microbiota analysis and metabolomics to clarify the mechanism of the effects of curcumol against liver fibrosis.

\section{Materials And Methods}

\section{Animals and procedures}

Male C57BL/6J mice (body weight, 20-25 g) were purchased from Hunan SJA Laboratory Animal Co, Ltd. (Changsha, China). All of the animals were raised in Guangxi University of Chinese Medicine (Nanning, China) after the experimental procedures received the approval of the institutional and local committees. Twenty-four mice were randomly divided into a blank control group, a model group, and a curcumol treatment group, with eight mice in each group. Mice in the model and curcumol groups were injected intraperitoneally with $40 \% \mathrm{CCl}_{4}$ (Tianjin Fuyu Fine Chemical Company, China) ( $\mathrm{CCl}_{4}$ : peanut oil, 2:3 preparation) at $5 \mathrm{~mL} / \mathrm{kg}$, twice a week, for a total of six weeks. The mice in the blank group were given the same amount of ordinary peanut oil. Curcumol (Shanghai Aladdin Chemical Co., Ltd., China) was administered to mice by gavage at $30 \mathrm{~mL} / \mathrm{kg}$ once a day for six weeks. The dosage of curcumol was based on the Chinese Pharmacopoeia and the results of previous studies [20]. The mice in the blank group and the model group were given normal saline for six weeks. All of the animals were fasted for 24 $\mathrm{h}$ at the end of the experiment, and then blood, liver, and intestinal contents were sampled.

\section{Liver histopathology}


The liver tissue was fixed in 10\% neutral buffered formalin and embedded in paraffin. Hematoxylin and eosin (H\&E) (Sigma, St. Louis, MO, USA) staining was used for pathological evaluation. Masson staining was used to assess collagen. The photos were taken under a microscope (Olympus CX23, Japan).

\section{Serum biochemistry}

Blood samples were allowed to stand for one hour after being taken from the eyelids of mice, and serum was extracted after centrifugation. The serum levels of aspartate aminotransferase (AST), alanine

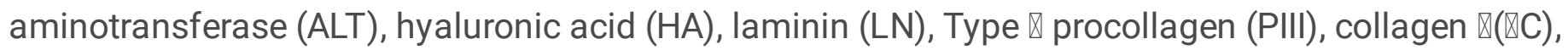
tumor necrosis factor-a (TNF-a), interleukin 6 (IL-6), and interleukin $1 \beta$ (IL-1 $\beta)$ were measured using ELISA kits (Shanghai Enzyme Link Biotechnology Co., Ltd, China)[45].

\section{Measurement of liver hydroxyproline content}

Liver hydroxyproline content was determined by the alkaline hydrolysis method as described in the assay kit (Nanjing Jiancheng Bioengineering Institute, Nanjing, China).

\section{Quantitative reverse transcription}

Total RNA of liver tissue was extracted using the Trizol reagent (Sigma, St. Louis, MO, USA) and reversetranscribed to cDNA by reverse transcriptase (Promega, USA) following the manufacturer's instructions. A 20-mL reaction volume (10 mL of SYBR Green PCR Master Mix [Takara, China], $300 \mathrm{nM}$ primer, $0.4 \mathrm{~mL}$ of Dyell, and $2 \mathrm{~mL}$ of cDNA product) was employed using an ABI 7500 Real-Time PCR System (ABI, Foster, CA, USA). The amplification reactions were performed as follows: one cycle at $95^{\circ} \mathrm{C}$ for $30 \mathrm{~s}, 60^{\circ} \mathrm{C}$ for 15 $\mathrm{s}$, and then $95^{\circ} \mathrm{C}$ for $15 \mathrm{~s}$ followed by 40 cycles of $95^{\circ} \mathrm{C}$ for $10 \mathrm{~s}$ and $60^{\circ} \mathrm{C}$ for $32 \mathrm{~s}$. Dissolution curve analysis was performed after each cycle at $95^{\circ} \mathrm{C}$ for $15 \mathrm{~s}$ and at $60^{\circ} \mathrm{C}$ for $60 \mathrm{~s}$. The fold changes were calculated using the comparative $2^{-\triangle \triangle C T}$ method. The sequences of primers used in this study were as follows: TLR4, Forward primer:5'-AGGGTTTCCTGTCAGTATCAAGTTT-3', Reverse primer: 5'TGATGCCTCCCTGG

CTCCT-3'; NF-KB, Forward primer:5'-TGACGGGAGGGGAAGAAATC-3', Reverse primer: 5'TGAACAAACACGGAAGCTGG-3'; $\beta$-actin, Forward primer: 5'-ATCACTATTGGCAACGAGCGGTTC-3', Reverse primer: 5'-CAGCACTGTGTTGGCATAGAGGTC-3'.

\section{Immunohistochemistry}

Paraffin-embedded liver tissues were cut into 3- $\mu$ m sections. After deparaffinization, sections were subjected to antigen recovery, blocked, and then incubated with rabbit anti-TLR4 (1:1000) and anti-NFKB p65 (1:500) antibodies (Abcam, UK) at $4^{\circ} \mathrm{C}$ overnight. Diaminobenzidine (Sigma, St. Louis, MO, USA) was used to detect the immunocomplex, whereas hematoxylin was used for nuclear counterstaining. A light microscope (Olympus CX23, Japan) was used to capture the immunohistochemical TIFF images. TLR4 and NF-KB were quantitatively analyzed by ImageJ software (V6.0 National Institutes of Health) [46]. 


\section{Fecal DNA extraction and high-throughput 16S rRNA sequencing}

Mouse feces were collected for $4 \mathrm{~h}$ and immediately kept at $-80^{\circ} \mathrm{C}$ until DNA extraction. Microbial DNA was extracted from 30 gut content (stool) samples using the E.Z.N.A. stool DNA Kit (Omega Bio-tek, Norcross, GA, USA) according to the manufacturer's protocol. The 16S rDNA V3-V4 region of the eukaryotic ribosomal RNA gene was amplified by PCR $\left(95^{\circ} \mathrm{C}\right.$ for 2 min followed by 27 cycles at $98^{\circ} \mathrm{C}$ for $10 \mathrm{~s}, 62^{\circ} \mathrm{C}$ for $30 \mathrm{~s}, 68^{\circ} \mathrm{C}$ for $30 \mathrm{~s}$, and a final extension at $68^{\circ} \mathrm{C}$ for $10 \mathrm{~min}$ ) using the above primers, where the barcode was an eight-base sequence unique to each sample. PCR reactions were performed in triplicate $50-\mu \mathrm{L}$ mixtures containing $5 \mu \mathrm{L}$ of $10 \times \mathrm{KOD}$ Buffer, $5 \mu \mathrm{L}$ of $2.5 \mathrm{mM}$ dNTPs, $1.5 \mu \mathrm{L}$ of each primer $(5 \mu \mathrm{M}), 1 \mu \mathrm{L}$ of KOD Polymerase, and $100 \mathrm{ng}$ of template DNA.

Amplicons were extracted from $2 \%$ agarose gels and purified using an AxyPrep DNA Gel Extraction Kit (Axygen Biosciences, Union City, CA, USA) according to the manufacturer's instructions and quantified using QuantiFluor-ST (Promega, USA). Purified amplicons were pooled at equimolar ratios and paired-end sequenced $(2 \times 250)$ on an Illumina platform according to the standard protocols[47].

\section{Serum metabolomics}

After thawing at room temperature, a pipette was used to transfer $100 \mathrm{~mL}$ of plasma to a $1.5-\mathrm{mL}$ EP tube. Methanol (300 mL) (Thermo fisher, USA) was added to precipitate the proteins in the serum, followed by adding $10 \mathrm{~mL}$ internal standard $(2.9 \mathrm{mg} / \mathrm{mL}$ 2-chlorophenylalanine). After vortexing and mixing for $30 \mathrm{~s}$, the sample was centrifuged at $4^{\circ} \mathrm{C}$ for 15 minutes at a speed of $12,000 \mathrm{rpm} ; 200 \mathrm{ml}$ of the supernatant was then transferred to a sample vial for subsequent testing. The instrument analysis platform was an LC-MS system (Thermo, Ultramate 3000LC, Orbitrap Elite) and a C18 chromatographic column (Hypergod $\mathrm{C} 18$ [100 $\times 2.1 \mathrm{~mm} \times 1.9 \mu \mathrm{m}])($ Thermo Fisher, USA). The chromatographic separation conditions were as follows: the flow rate was $0.3 \mathrm{~mL} / \mathrm{min}$; the composition of mobile phase A was water (Merck, Germany) + $0.1 \%$ formic acid (Thermo Fisher, USA). The composition of mobile phase B was acetonitrile $+0.1 \%$ formic acid; the injection volume was $4 \mu \mathrm{L}$, and the autosampler temperature was $4^{\circ} \mathrm{C}$.

For the metabonomics detection of blood samples, the parameters of the positive ion mode were as follows: the heater temperature was $300^{\circ} \mathrm{C}$; the gas flow rate was 45 arb; the auxiliary gas flow rate was $15 \mathrm{arb}$; the exhaust gas flow rate was I amb, and the electrospray voltage was $3.0 \mathrm{KV}$. The capillary temperature was $350^{\circ} \mathrm{C}$, and the S-lens RF level was $30 \%$. For the metabonomics detection of blood samples, the negative ion mode parameters were as follows: the heater temperature was $300^{\circ} \mathrm{C}$; the sheath gas flow rate was 45 arb; the auxiliary gas flow rate was 15 arb; the exhaust gas flow rate was I arb; the electrospray voltage was $3.2 \mathrm{KV}$; the capillary temperature was $350^{\circ} \mathrm{C}$, and the S-lens RF level was $60 \%[48]$.

\section{Statistical Analysis}

Data analysis was processed by GraphPad Prism 7 software (GraphPad Software Inc., San Diego, CA). The data that conformed to a normal distribution were represented by the mean \pm standard deviation. If 
the data were normally distributed and the variance was homogeneous, the $t$-test was used. If the above conditions were not met, the Mann-Whitney Unonparametric test was used; the count data employed the chi-squared test. The biological databases HMDB (https://hmdb.ca/) and KEGG (https://www.kegg.jp/) were employed for biomarker identification and metabolic pathways and gene function analysis. Pearson's correlation analysis was used for bivariate correlation.

\section{Results}

\section{Curcumol attenuates liver fibrosis in mice}

We examined the effect of curcumol in vivo using male mice with $\mathrm{CCl}_{4}$-induced liver fibrosis (Figure 1A). Different histological analyses were used to assess liver fibrosis. Masson and H\&E staining showed that the liver damage induced by $\mathrm{CCl}_{4}$ displayed heavier collagen deposition and structural disorders accompanied by inflammation. Curcumol had the effect of reducing liver fibrosis (Figure 1B). These results were based on serum AST and ALT (Figure 1C), and serum fibrosis HA, LN, PC区, and $\triangle-C$ indicators (Figure 1D). Liver hydroxyproline content (Figure 1E) was also verified.

\section{Curcumol inhibits the activity of the TLR4/NF-KB signaling pathway}

We found through RT-PCR and immunohistochemistry experiments that curcumol inhibited the expression of TLR4 and NF-KB at the gene and protein levels (Figures 2A, 2B). Because TLR4 and NF-KB are key molecules in the TLR4 signaling pathway, inhibiting their expression will affect the expression of downstream signaling molecules. The TLR4/NF-KB signaling pathway is a classic pathway that regulates the body's inflammatory response, and thus inhibiting the activity of this signaling pathway will inhibit the inflammatory response. Through the ELISA experiment, we found that curcumol inhibited the expression of inflammatory factors (Figure $2 \mathrm{C}$ ), further verifying that curcumol could inhibit the inflammatory response in mice with liver fibrosis.

\section{Effect of curcumol on the gut microbiota of mice with liver fibrosis induced by $\mathrm{CCl}_{4}$}

The 16S rRNA genes of 24 fecal samples were amplified by PCR and then subjected to high-throughput sequencing. The high-throughput sequencing obtained 2,163,264 reads. Under the condition of $97 \%$ similarity, the reads were clustered into operational taxonomic units (OTUs) of classified species. A total of 1903 OTUs were obtained (Figure 3A). The sequencing coverage indexes of the three groups were all greater than 0.99 (Figure 3B). The results indicated that the sample groups that were not tested had a low sequence probability.

We analyzed the diversity of the gut microbiota of the sample (alpha diversity). The evaluation indicators were the Chao1 index, the Observed species index, Shannon's index, and Simpson's index. The Chao 1 index, the Observed species index, and Shannon's index are positively correlated with sample species richness, while Simpson's index is the opposite, being negatively correlated with sample species richness. In this study, the Chao 1, Observed species, Shannon and Simpson indexes showed that after 
$\mathrm{CCl}_{4}$ induction, the diversity of the gut microbiota of mice increased, but after the intervention of curcumol, the diversity of the gut microbiota of mice decreased (Figure $3 \mathrm{C}$ ). At the same time, the similarity of the intestinal flora of the samples was analyzed (beta diversity). The main evaluation methods were PCoA and NMDS analyses, both of which reduce the complexity of the data without changing the main characteristics, thereby effectively extracting the most important features of the data. The analysis is designed to reveal the core characteristic changes hidden within the larger dataset. In the PCoA and NMDS analysis graphs, the gap between different samples is represented by the distance between the coordinate axes. The closer the distance, the higher the sample similarity, and the greater the distance, the lower the sample similarity. PCoA and NMDS analysis clearly separated the blank, model, and curcumol groups, and there were differences in the composition of the intestinal flora (Figure 3D).

Comparing the structural changes of intestinal flora in each group based on OTUs, at the phylum level (Figure 4A), Bacteroidota, Firmicutes, Proteobacteria, Campylobacterota, Deferribacteres,

Desulfobacterota, Actinobacteriota, unidentified_Bacteria, Cyanobacteria, and Acidobacteriota had higher abundances. The results indicate that these flora play important roles in each group. At the same time, we conducted a statistical analysis of the bacterial population for taxa with an abundance greater than $10 \%$ and found that $\mathrm{CCl}_{4}$-induced liver fibrosis increased the abundance of Bacteroidota and decreased the abundance of Firmicutes and Proteobacteria, and curcumol treatment reduced the abundance of Bacteroidota. The abundances of Firmicutes and Proteobacteria were increased. At the genus level (Figure 4B), the higher abundance of Bacteroides, Acinetobacter, Staphylococcus, Helicobacter, Lachnospiraceae_NK4A136_group, Lactobacillus, Empedobacter, Alloprevotella, Escherichia-Shigella, Candidatus_Arthromitus, and Candidatus_Arthromitus indicate that these flora play an important role in each group. At the same time, we conducted a statistical analysis of the bacterial population for taxa with an abundance greater than $10 \%$ and found that $\mathrm{CCl}_{4}$-induced liver fibrosis increased the abundance of Bacteroides and Acinetobacter and decreased the abundance of Staphylococcus. The curcumol treatment reduced the abundance of Bacteroides, and the abundances of Acinetobacter and Staphylococcus were increased.

An LEfSe analysis was performed to further characterize the distinguishing phylotypes in the gut microbiota of the different groups. At the same time, a linear discriminant analysis (LDA) was performed to estimate how the abundance of each species (component) affected the differences between groups. Microorganisms with different LDA scores greater than the set value (set value $=2$ ) were considered as biomarkers with statistically significant differences. Figure 4 shows the microbial flora with significant differences in abundance in the three groups, and the length of the histogram represents the impact of significantly different species. Prevotellaceae, Negativicutes, Eubacteriales, and Eubacteriaceae had higher expression in the blank group, while Acinetobacter, Empedobacter, Weeksellaceae, Sphingobacterium, and Alcaligenaceae had higher expression in the model group, and Veillonellaceae, Prerotella_oulorum, and Alistipes_finegoldii had higher expression in the curcumol group (Figure 4C). The results of the study show that after $\mathrm{CCl}_{4}$ induced liver fibrosis in mice, the structure of the mouse fecal flora underwent significant changes, and curcumol could adjust or improve these changes. The results also show that the intestinal flora plays an important role in the prevention and treatment of liver fibrosis. 


\section{Effect of curcumol on metabolites of mice with liver fibrosis induced by $\mathrm{CCl}_{4}$}

Principal component analysis (PCA) was used to perform data analysis on quality control samples. In the PCA score chart, each point represents a sample, and the closer the metabolites contained in the sample, the more compact the sample points in the figure. In the principal component analysis of the quality control samples in the positive and negative ion modes, the quality control samples (purple) were tightly clustered and were clearly separated from the experimental samples (Figures $5 A-B$ ), indicating that the data were of good quality and that the instrument was stable.

In order to obtain biomarkers of liver fibrosis, we used Partial Least Squares Discriminant Analysis (PLSDA) to analyze the differences among metabolites between the model group and the blank group. Significant differences were found in the positive ion PLS-DA ( $R 2=1.00, \mathrm{Q} 2=0.91)$ Most of the samples were separated, indicating that there were differences among the levels of metabolites between the two groups (Figures $5 C-D$ ); in the negative ion PLS-DA analysis (R2=0.99, Q2 $=0.87$ ), most of the samples were separated (Figures 5E-F). Based on the Variable Importance in the Projection (VIP) value [21], multivariate analysis along with the $\mathrm{P}$ value and the multiple of difference were used to screen for differentially expressed metabolites. The screening criteria were a P-value $<0.05 ; \mathrm{VIP} \geq 1$, and fold change $\leq 0.833$ or $\geq 1.2$ [22] (Table 1). The PLS-DA for the metabolic differences between the curcumol group and the model group (Figure 5) revealed that curcumol regulated nine metabolic pathways (Figure 5G).

Table.1 Identified potential biomarkers in $\operatorname{serum}(\mathrm{n}=8)$ 


\begin{tabular}{|c|c|c|c|c|c|c|c|c|}
\hline bolite & $\mathrm{FC}$ & VIP & $\begin{array}{l}\mathrm{Up} / \\
\text { Down }\end{array}$ & No. & Metabolite & $\overline{\mathrm{FC}}$ & $\overline{\mathrm{VIP}}$ & $\begin{array}{l}\mathrm{Up} / \\
\text { Down }\end{array}$ \\
\hline aionine & 0.60 & 1.67 & down & 28 & Propionylcarnitine & 1.57 & 1.86 & up \\
\hline гotriene C4 & 0.11 & 1.78 & down & 29 & $12,13-\mathrm{EODE}$ & 2.20 & 1.59 & up \\
\hline saponin I & 2.31 & 1.88 & up & 30 & N-Methylhydantoin & 2.59 & 2.06 & up \\
\hline Jorvaline & 0.43 & 1.92 & down & 31 & 4-Hexyloxyaniline & 1.91 & 1.57 & up \\
\hline & 0.20 & 1.45 & down & 32 & Methionine sulfoxide & 1.38 & 1.07 & up \\
\hline $\begin{array}{l}\text { utyryigiycine } \\
\text { sphylline }\end{array}$ & 0.55 & 1.59 & down & 33 & $\begin{array}{l}\text { 5-[(10Z)-14-(3,5- } \\
\text { dihydroxyphenyl)tetradec-10- } \\
\text { en-1-yl]benzene-1,3-diol }\end{array}$ & 0.25 & 1.45 & down \\
\hline a-D-Ala & 0.49 & 1.08 & down & 34 & Trimethyllysine & 0.57 & 1.37 & down \\
\hline droroseoside & 0.41 & 1.14 & down & 35 & Asp-glu & 2.19 & 2.04 & up \\
\hline Irginine & 0.43 & 1.26 & down & 36 & $\alpha$-Lapachone & 3.02 & 1.96 & up \\
\hline $16: 0 / 22: 6)$ & 0.61 & 1.27 & down & 37 & Pantethine & 4.19 & 1.35 & up \\
\hline PC 18:0 & 0.35 & 1.68 & down & 38 & LPE $22: 4$ & 0.27 & 1.50 & down \\
\hline $14: 1 \mathrm{e} / 24: 1)$ & 2.25 & 1.97 & up & 39 & O-Aceyl-L-Serine & 2.11 & 1.83 & up \\
\hline $22: 6$ & 0.53 & 2.09 & down & 40 & $\begin{array}{l}\text { 3-hydroxy-2-(3-nitro-4- } \\
\text { piperidinobenzyl)propanenitrile }\end{array}$ & 2.96 & 1.39 & up \\
\hline gulsterone & 2.49 & 2.00 & up & 41 & $\mathrm{PC}(4: 0 / 18: 5)$ & 2.81 & 1.92 & up \\
\hline eidopropionic & 2.91 & 2.10 & up & 42 & PC $(18: 3 e / 3: 0)$ & 5.03 & 1.97 & up \\
\hline Lamoylglycine & 0.15 & 1.61 & down & 43 & $\begin{array}{l}\mathrm{N} \sim 5 \sim-(1,3,5 \text {-trimethyl-1H- } \\
\text { pyrazol-4-yl)-1 H-1 } 2,4 \text {-triazole- } \\
\text { 3,5-diamine }\end{array}$ & 1.49 & 2.04 & up \\
\hline sthylguanine & 1.53 & 2.24 & up & 44 & Histamine & 2.34 & 1.81 & up \\
\hline glutamic acid & 0.13 & 1.58 & down & 45 & Gly-Tyr & 1.72 & 1.84 & up \\
\hline Čarnitine & 1.40 & 1.77 & up & 46 & MÄG (18:2) & 6.18 & 1.90 & up \\
\hline & 0.17 & 2.32 & down & 47 & DNH & 2.48 & 1.78 & up \\
\hline $\begin{array}{l}\text { setyl-L- } \\
\text { amic acid }\end{array}$ & 1.56 & 1.40 & up & 48 & 2-Deoxyuridine & 1.69 & 1.47 & up \\
\hline benzylidene- & 1.98 & 1.97 & up & 49 & $\begin{array}{l}\text { 4-methyl-5-oxo-2-pentyl-2,5- } \\
\text { dihydrofuran-3-carboxylic acid }\end{array}$ & 2.35 & 1.61 & up \\
\hline $\begin{array}{l}\text { b-Dihydroxy- } \\
\text { drosten-17- }\end{array}$ & 3.32 & 2.04 & up & 50 & Cytidine & 1.88 & 1.59 & up \\
\hline oretinol & 6.09 & 2.04 & up & 51 & Valproic acid & 2.44 & 1.86 & up \\
\hline $\begin{array}{l}\text { ) }-9,10,11- \\
\text { droxyoctadec- }\end{array}$ & 2.90 & 1.71 & up & 52 & 6-Keto-prostaglandin f1alpha & 2.62 & 1.71 & up \\
\hline $\begin{array}{l}\text { noic acid } \\
\text { etyl-11-keto- } \\
\text { swellic acid }\end{array}$ & 3.46 & 1.98 & up & 53 & $\mathrm{PC}(14: 1 \mathrm{e} / 3: 0)$ & 1.54 & 1.88 & up \\
\hline adyne 1 - & 2.01 & 1.69 & up & 54 & indole-5,6-quinone & 1.69 & 1.46 & up \\
\hline
\end{tabular}

Notes:FC:fold-change;VIP:variable importance in the projection.

\section{Correlation between gut microbiota and metabolites}

Correlation analysis of metabolites with significant differences between the model group and the blank group and the intestinal flora showed that cytidine was significantly related to Bacteroidota and Proteobacteria at the phylum level (Figure 6A). At the genus level, Bacteroides was significantly correlated with 3-ureidoproplonic acid and methionine sulfoxide, and valproic acid and Staphylococcuus were significantly correlated (Figure 6B). Correlation analysis of metabolites with significant differences between the model group and the curcumol group and the intestinal flora showed that Bacteroidota and prostaglandin B2 were significantly correlated at the phylum level (Figure 6C). At the genus level, Bacteroides and dopaquinone were significantly correlated (Figure 6D). 


\section{Discussion}

Liver fibrosis is a wound healing process accompanying repeated chronic liver injury [23]. It can accelerate the development of chronic liver disease by destroying normal liver parenchyma, eventually leading to liver cirrhosis, liver failure, and even primary liver cancer [24]. Although the pathogenesis of liver fibrosis that includes inflammation, activation of hepatic stellate cells, and formation of an extracellular matrix has been widely recognized, effective and powerful treatments have not yet been obtained [25]. Therefore, it is necessary to develop a high-efficiency, low-toxicity, multi-target treatment tool for liver fibrosis. We have combined gut microbiota analysis and metabolomics to investigate the pathogenesis of liver fibrosis and to elucidate the mechanism of action of curcumin against liver fibrosis, thereby providing a theoretical basis for its clinical use. It has been verified at the animal level that curcumol can improve liver pathological changes and liver function. Molecular biology experiments have found that curcumol can reduce the expression of downstream inflammatory factors by inhibiting the activity of the TLR4/NF-KB signaling pathway. Lipopolysaccharide (LPS), the main component of the cell wall of Gram-negative bacteria, is the ligand of TLR4. The combination of the two promotes the nucleation of the inflammatory transcription factor NF-KB and triggers the massive release of inflammatory cytokines such as IL-6, TNFa, and IL-8 to enhance the inflammatory response of the liver and form an "inflammatory waterfall" that ultimately leads to the formation of liver fibrosis [26-27].

We have studied the gut microbes of mice with liver fibrosis and found that the diversity and abundance of fecal microbes in mice with liver fibrosis were significantly different from those in the control groups. At the phylum level, the abundances of Bacteroidota, Firmcutes, and Proteobacteria were significantly different among the three groups. At the genus level, the abundances of Bacteroides, Acinetobacter, and Staphylococcus were significantly different between the three groups. Both Bacteroidota and Bacteroides were highly expressed in the model group, consistent with the results of previous studies [28]. Studies have also confirmed that the abundance of Firmcutes will decrease when liver fibrosis occurs [29]. Acinetobacter is an important pathogen of tissue infection [30]. We found that Acinetobacter, Empedobacter, Weeksellaceae, Sphingobacterium, and Alcaligenaceae were present in the model group. Acinetobacter plays an important role in death caused by bacteremia in patients with liver cirrhosis [31]. Empedobacter has a significant increase in abundance in irritable bowel syndrome after inflammation and is significantly related to IL-8 expression, indicating that Empedobacter plays an important role in inflammatory diseases; previous studies have shown that long-term high-fat, high-sugar, and highcholesterol diets can cause experimental pigs to develop liver steatosis and liver fibrosis. The abundance of Empedobacter was significantly increased in the cecal microbiota of experimental pigs [32-33]. In a study of hepatic encephalopathy, it was found that Alcaligenaceae strengthened the intestine-liver-brain connection that is closely related to changes in cognitive function [34]. At the same time, we found that Veillonellaceae, Prerotella_oulorum, and Alistipes_finegoldii played important roles in the curcumol group that may be related to curcumol in the prevention and treatment of liver fibrosis. In non-alcoholic fatty liver fibrosis, Veillonellaceae is the main microbial group, and in this study Veillonellaceae was present in the liver. Sclerosis plays an important role in cognitive dysfunction, and it is positively correlated with the expression of inflammatory factors [35-36]. Alistipes_finegoldii is a relatively new subclade of the 
phylum Bacteroidetes that is mainly isolated from medical clinical samples. Compared with other members of the phylum Bacteroidetes, its isolation rate is lower. Studies have found that Alipipes_finegoldii is reduced throughout the process of liver fibrosis, and that the decrease of Alipipes_finegoldii leads to decompensation of liver cirrhosis. In addition, the decrease of Alipipes_finegoldii abundance can lead to the occurrence of liver cirrhosis and hepatic encephalopathy [37-39].

Different groups of non-targeted metabolite profiles can not only help us identify specific metabolites but also can provide clues to the mechanism of action of curcumol. We found 54 metabolites that were significantly different between the model group and the blank group based on the screening conditions. The enrichment analysis of metabolites between the curcumol group and the model group revealed that pyrimidine metabolism, tyrosine metabolism, beta-alanine metabolism, pantothenate and CoA biosynthesis, glutathione metabolism, porphyrin and chlorophyll metabolism, arachidonic acid metabolism, primary bile acid, and steroid hormone biosynthesis differed between groups. These metabolic pathways are closely related to curcumol's effects on liver fibrosis. Tyrosine is the precursor component of the synthesis of a variety of vasoactive substances in the human body. Studies have found that vasoactive substances play an important role in abnormal hepatic vascular beds and their resulting influence on hepatic circulation. The structure of blood vessels in the liver is abnormal in such cases, and the capillaries of the liver sinuses form sinusoids. The increase in peripheral blood flow resistance will cause the liver to be in a state of high resistance and aggravate the degree of liver fibrosis [40]. Coenzyme A ( $\mathrm{CoA}$ ) and its degradation product, pantothenic acid, play important roles in the physiological and pathological processes of the liver [41]. Studies have shown that Radix Gentianae can improve liver function by regulating the metabolic pathways of porphyrin and chlorophyll, thereby exerting a metabolic protective effect on the liver [42]. Arachidonic acid is important in the inflammatory metabolic network and is a substance with strong biological activity related to oxidative stress. Under oxidative stress conditions, the conversion of linoleic acid to arachidonic acid in the body will be enhanced; many unsaturated fatty acids will be produced during the metabolism of arachidonic acid, and these substances are powerful inflammatory mediators [43]. Studies have shown that liver damage caused by chronic liver disease leads to a decrease in the liver's clearance of bile acids, and ultimately an increase in serum bile acid levels. Bile acids promote liver damage by regulating the induced apoptosis and necrosis of hepatocytes, and ultimately promote the occurrence of cirrhosis or liver failure [44]. Steroids are indispensable and important hormones for life-sustaining activities. The body's steroids secreted by the adrenal glands can participate in the regulation of blood sugar, fats, and other aspects of metabolism, thereby maintaining the body's normal physiological operations.

In the correlation analysis of intestinal flora and metabolites, the correlation between Bacteroidota and Proteobacteria and cytidine, and the correlation between Bacteroides and 3-ureidoproplonic acid and methionine sulfoxide may play important roles in the formation of liver fibrosis. Curcumol exerts an antihepatic fibrosis effect by regulating the relative abundance of Bacteroidota and Bacteroides and by participating in the metabolism of prostaglandin B2, oleoyl ethanolamide, isoralerylglycine, cytidine, and Asp-glu. This may be related to reducing the expression of inflammatory factors and improving liver 
inflammation (Figure 7). The aim of this study was to determine whether curcumol could improve liver fibrosis by regulating intestinal flora and metabolites. However, due to funding and time constraints, there are still some shortcomings: the selected sample size was small; only a correlation study was carried out, and only the $\mathrm{CCl}_{4}$ induced liver fibrosis model has been studied.

\section{Conclusions}

A total of 54 differentially expressed metabolites, including 4-oxoretinol, may be potential biomarkers of liver fibrosis. Curcumol regulates the abundance of Bacteroidota and Bacteroides and participates in the activities of nine metabolic pathways, thereby inhibiting the activity of the TLR4/NF-KB signaling pathway, reducing the secretion of downstream inflammatory factors, and improving liver inflammation. These results may help to further characterize the pharmacological mechanism of curcumol against liver fibrosis.

\section{Declarations}

\section{Authors' contributions}

YZ and TZ designed the study, YZ and JW wrote the manuscript, JW, JRand TL conducted this research. All authors have read and approved the manuscript.

\section{Funding}

This study was funded by the National Natural Science Foundation of China (Grant Nos. 81960751, 81960761, and 81660705), the Guangxi Natural Science Foundation Youth Project (Grant No. 2020GXNSFBA297094), the Guangxi Young and Middle-aged Teachers' Research Ability Improvement Project (Grant No. 2020KY59009), the Guangxi Zhuangyao Pharmaceutical Key Laboratory (Grant Nos. GXZYZZ2019-1, GXZYZZ2020-07), and the Guangxi University of Traditional Chinese Medicine Schoollevel Project Youth Fund (Grant No. 2020QN006).

\section{Availability of data}

The data that support the findings of this study are available from the corresponding author upon reasonable request.

\section{Ethical approval}

The study was approved by Animal Care and Use Committee of the Guangxi University of Traditional Chinese Medicine(2016-12-02-1).

\section{Competing interests}

The authors declare that there are no financial conflicts of interest. 
Acknowledgments

The authors wish to thank the Guangxi University of Chinese Medicine (Guangxi, China) for providing laboratory equipment and technological support.

\section{References}

[1]Roehlen N, Crouchet E, Baumert TF. Liver Fibrosis: Mechanistic Concepts and Therapeutic Perspectives. Cells. 2020;9(4):875.

[2]Zhou WC, Zhang QB, Qiao L. Pathogenesis of liver cirrhosis. World J Gastroenterol. 2014;20(23):73127324.

[3]Seki E, Brenner DA. Recent advancement of molecular mechanisms of liver fibrosis. J Hepatobiliary Pancreat Sci. 2015;22(7):512-518.

[4]Sender R, Fuchs S, Milo R. Are We Really Vastly Outnumbered? Revisiting the Ratio of Bacterial to Host Cells in Humans. Cell. 2016 Jan 28;164(3):337-340.

[5]Tripathi A, Debelius J, Brenner DA, Karin M, Loomba R, Schnabl B, Knight R. The gut-liver axis and the intersection with the microbiome. Nat Rev Gastroenterol Hepatol. 2018;15(7):397-411.

[6]Tilg H, Cani PD, Mayer EA. Gut microbiome and liver diseases. Gut. 2016;65(12):2035-2044.

[7]Paik YH, Schwabe RF, Bataller R, Russo MP, Jobin C, Brenner DA. Toll-like receptor 4 mediates inflammatory signaling by bacterial lipopolysaccharide in human hepatic stellate cells. Hepatology. 2003;37(5):1043-1055.

[8]Zhu Q, Zou L, Jagavelu K, Simonetto DA, Huebert RC, Jiang ZD, DuPont HL, Shah VH. Intestinal decontamination inhibits TLR4 dependent fibronectin-mediated cross-talk between stellate cells and endothelial cells in liver fibrosis in mice. J Hepatol. 2012;56(4):893-899.

[9]Yu J, He JQ, Chen DY, Pan QL, Yang JF, Cao HC, Li LJ. Dynamic changes of key metabolites during liver fibrosis in rats. World J Gastroenterol. 2019 Feb 28;25(8):941-954.

[10]Zhao L, Dong M, Liao S, Du Y, Zhou Q, Zheng H, Chen M, Ji J, Gao H. Identification of key metabolic changes in renal interstitial fibrosis rats using metabonomics and pharmacology. Sci Rep. 2016 Jun 3;6:27194.

[11]Dai D, Gao Y, Chen J, Huang Y, Zhang Z, Xu F. Time-resolved metabolomics analysis of individual differences during the early stage of lipopolysaccharide-treated rats. Sci Rep. 2016 Oct 3;6:34136.

[12]Cao H, Zhang A, Sun H, Zhou X, Guan Y, Liu Q, Kong L, Wang X. Metabolomics-proteomics profiles delineate metabolic changes in kidney fibrosis disease. Proteomics. 2015 Nov;15(21):3699-3710. 
[13]Ressom HW, Xiao JF, Tuli L, Varghese RS, Zhou B, Tsai TH, Ranjbar MR, Zhao Y, Wang J, Di Poto C, Cheema AK, Tadesse MG, Goldman R, Shetty K. Utilization of metabolomics to identify serum biomarkers for hepatocellular carcinoma in patients with liver cirrhosis. Anal Chim Acta. 2012 Sep 19;743:90-100.

[14]Tokushige K, Hashimoto E, Kodama K, Tobari M, Matsushita N, Kogiso T, Taniai M, Torii N, Shiratori K, Nishizaki Y, Ohga T, Ohashi Y, Sato T. Serum metabolomic profile and potential biomarkers for severity of fibrosis in nonalcoholic fatty liver disease. J Gastroenterol. 2013 Dec;48(12):1392-400.

[15]Zhang Y, Zhao M, Liu Y, Liu T, Zhao C, Wang M. Investigation of the therapeutic effect of Yinchen Wuling Powder on CCl4-induced hepatic fibrosis in rats by $1 \mathrm{H}$ NMR and MS-based metabolomics analysis. J Pharm Biomed Anal. 2021 Jun 5;200:114073.

[16]Chang H, Meng HY, Liu SM, Wang Y, Yang XX, Lu F, Wang HY. Identification of key metabolic changes during liver fibrosis progression in rats using a urine and serum metabolomics approach. Sci Rep. 2017 Sep 12;7(1):11433.

[17]Zhou Y, Wu R, Cai FF, Zhou WJ, Lu YY, Zhang H, Chen QL, Su SB. Xiaoyaosan decoction alleviated rat liver fibrosis via the TGF $\beta /$ Smad and Akt/FoxO3 signaling pathways based on network pharmacology analysis. J Ethnopharmacol. 2021 Jan 10;264:113021.

[18]Rezzani R, Franco C, Rodella LF. Curcumin as a Therapeutic Strategy in Liver Diseases. Nutrients. 2019 Oct 17;11(10):2498.

[19]Li N, Liu TH, Yu JZ, Li CX, Liu Y, Wu YY, Yang ZS, Yuan JL. Curcumin and Curcumol Inhibit NF-kB and TGF- $\beta 1 /$ Smads Signaling Pathways in CSE-Treated RAW246.7 Cells. Evid Based Complement Alternat Med. 2019 Mar 17;2019:3035125.

[20]Zheng Y, Wang JR, Liu LL, Wang JH, Zhao TJ. To study the molecular mechanism of curcumol against liver fibrosis based on Toll-like receptor 4/nuclear factor-kB signaling pathway. Chin J Clin Hepatol. 2020,36(07):1508-1513.

[21]Heischmann, S. et al. Exploratory Metabolomics Profiling in the Kainic Acid Rat Model Reveals Depletion of 25-Hydroxyvitamin D3 during Epileptogenesis. Scientific Reports. 6 (2016).

[22]Sreekumar, A. et al. Metabolomic profiles delineate potential role for sarcosine in prostate cancer progression. Nature. 457,910- 914 (2009).

[23]Xie H, Su D, Zhang J, Ji D, Mao J, Hao M, Wang Q, Yu M, Mao C, Lu T. Raw and vinegar processed Curcuma wenyujin regulates hepatic fibrosis via bloking TGF- $\beta /$ Smad signaling pathways and upregulation of MMP-2/TIMP-1 ratio. J Ethnopharmacol. 2020 Jan 10;246:111768.

[24]Lei Y, Wang QL, Shen L, Tao YY, Liu CH. MicroRNA-101 suppresses liver fibrosis by downregulating PI3K/Akt/mTOR signaling pathway. Clin Res Hepatol Gastroenterol. 2019 Oct;43(5):575-584. 
[25]Schuppan D, Ashfaq-Khan M, Yang AT, Kim YO. Liver fibrosis: Direct antifibrotic agents and targeted therapies. Matrix Biol. 2018 Aug;68-69:435-451.

[26]Teratani T, Tomita K, Suzuki T, Oshikawa T, Yokoyama H, Shimamura K, Tominaga S, Hiroi S, Irie R, Okada Y, Kurihara C, Ebinuma H, Saito H, Hokari R, Sugiyama K, Kanai T, Miura S, Hibi T. A highcholesterol diet exacerbates liver fibrosis in mice via accumulation of free cholesterol in hepatic stellate cells. Gastroenterology. 2012 Jan;142(1):152-164.

[27]Tang SC, Liao PY, Hung SJ, Ge JS, Chen SM, Lai JC, Hsiao YP, Yang JH. Topical application of glycolic acid suppresses the UVB induced IL-6, IL-8, MCP-1 and COX-2 inflammation by modulating NF-KB signaling pathway in keratinocytes and mice skin. J Dermatol Sci. 2017 Jun;86(3):238-248.

[28]Li MM, Zhou Y, Zuo L, Nie D, Li XA. Dietary fiber regulates intestinal flora and suppresses liver and systemic inflammation to alleviate liver fibrosis in mice. Nutrition. 2021 Jan;81:110959.

[29]Wan S, Nie Y, Zhang Y, Huang C, Zhu X. Gut Microbial Dysbiosis Is Associated With Profibrotic Factors in Liver Fibrosis Mice. Front Cell Infect Microbiol. 2020 Jan 31;10:18.

[30]Chao CM, Lai CC, Tang HJ, Ko WC, Hsueh PR. Skin and soft-tissue infections caused by Aeromonas species. Eur J Clin Microbiol Infect Dis. 2013 Apr;32(4):543-7.

[31]Liu CP, Chiang TT, Liu YM, Kuo SC, Yang YS, Lee YT, Chen TL, Shih SC; ACTION Study Group. A multicenter study on clinical characteristics of Acinetobacter bacteremia in patients with liver cirrhosis. J Microbiol Immunol Infect. 2019 Dec;52(6):956-965.

[32]Song YF, Pei LX, Chen L, Geng H, Yuan MQ, Xu WL, Wu J, Zhou JY, Sun JH. Electroacupuncture Relieves Irritable Bowel Syndrome by Regulating IL-18 and Gut Microbial Dysbiosis in a Trinitrobenzene Sulfonic Acid-Induced Post-Inflammatory Animal Model. Am J Chin Med. 2020;48(1):77-90.

[33]Panasevich MR, Meers GM, Linden MA, Booth FW, Perfield JW 2nd, Fritsche KL, Wankhade UD, Chintapalli SV, Shankar K, Ibdah JA, Rector RS. High-fat, high-fructose, high-cholesterol feeding causes severe NASH and cecal microbiota dysbiosis in juvenile Ossabaw swine. Am J Physiol Endocrinol Metab. 2018 Jan 1;314(1):E78-E92.

[34]Bajaj JS. The role of microbiota in hepatic encephalopathy. Gut Microbes. 2014 May-Jun;5(3):397403.

[35]Lee G, You HJ, Bajaj JS, Joo SK, Yu J, Park S, Kang H, Park JH, Kim JH, Lee DH, Lee S, Kim W, Ko G. Distinct signatures of gut microbiome and metabolites associated with significant fibrosis in non-obese NAFLD. Nat Commun. 2020 Oct 5;11(1):4982.

[36]Bajaj JS, Ridlon JM, Hylemon PB, Thacker LR, Heuman DM, Smith S, Sikaroodi M, Gillevet PM. Linkage of gut microbiome with cognition in hepatic encephalopathy. Am J Physiol Gastrointest Liver Physiol. 2012 Jan 1;302(1):G168-75. 
[37]Parker BJ, Wearsch PA, Veloo ACM, Rodriguez-Palacios A. The Genus Alistipes: Gut Bacteria With Emerging Implications to Inflammation, Cancer, and Mental Health. Front Immunol. 2020 Jun 9;11:906.

[38]lebba V, Guerrieri F, Di Gregorio V, Levrero M, Gagliardi A, Santangelo F, Sobolev AP, Circi S, Giannelli V, Mannina L, Schippa S, Merli M. Combining amplicon sequencing and metabolomics in cirrhotic patients highlights distinctive microbiota features involved in bacterial translocation, systemic inflammation and hepatic encephalopathy. Sci Rep. 2018 May 29;8(1):8210.

[39]Sung CM, Lin YF, Chen KF, Ke HM, Huang HY, Gong YN, Tsai WS, You JF, Lu MJ, Cheng HT, Lin CY, Kuo CJ, Tsai IJ, Hsieh SY. Predicting Clinical Outcomes of Cirrhosis Patients With Hepatic Encephalopathy From the Fecal Microbiome. Cell Mol Gastroenterol Hepatol. 2019;8(2):301-318.

[40]Liu S, Premont RT, Rockey DC. Endothelial nitric-oxide synthase (eNOS) is activated through G-proteincoupled receptor kinase-interacting protein 1 (GIT1) tyrosine phosphorylation and Src protein. J Biol Chem. 2014 Jun 27;289(26):18163-74.

[41]Naquet P, Kerr EW, Vickers SD, Leonardi R. Regulation of coenzyme A levels by degradation: the 'Ins and Outs'. Prog Lipid Res. 2020 Apr;78:101028.

[42]Xu S, Kong F, Sun Z, Xi Y, Qi F, Sun J. Hepatoprotective effect and metabonomics studies of radix gentianae in rats with acute liver injury. Pharm Biol. 2021 Dec;59(1):1172-1180.

[43]de Seymour JV, Conlon CA, Sulek K, Villas Bôas SG, McCowan LM, Kenny LC, Baker PN. Early pregnancy metabolite profiling discovers a potential biomarker for the subsequent development of gestational diabetes mellitus. Acta Diabetol. 2014 Oct;51(5):887-90.

[44]Zhou C, Jia HM, Liu YT, Yu M, Chang X, Ba YM, Zou ZM. Metabolism of glycerophospholipid, bile acid and retinol is correlated with the early outcomes of autoimmune hepatitis. Mol Biosyst. 2016;12(5):15741585.

[45]Kaminsky-Kolesnikov Y, Rauchbach E, Abu-Halaka D, Hahn M, García-Ruiz C, Fernandez-Checa JC, Madar Z, Tirosh O. Cholesterol Induces Nrf-2- and HIF-1a-Dependent Hepatocyte Proliferation and Liver Regeneration to Ameliorate Bile Acid Toxicity in Mouse Models of NASH and Fibrosis. Oxid Med Cell Longev. 2020;2020:5393761.

[46] Hu N, Guo C, Dai X, Wang C, Gong L, Yu L, Peng C, Li Y. Forsythiae Fructuse water extract attenuates liver fibrosis via TLR4/MyD88/NF-KB and TGF- $\beta$ /smads signaling pathways. J Ethnopharmacol. 2020;262:113275.

[47]Luo D, Chen K, Li J, Fang Z, Pang H, Yin Y, Rong X, Guo J. Gut microbiota combined with metabolomics reveals the metabolic profile of the normal aging process and the anti-aging effect of FuFang Zhenshu TiaoZhi(FTZ) in mice. Biomed Pharmacother. 2020;121:109550. 
[48]Li X, Xiao Y, Song L, Huang Y, Chu Q, Zhu S, Lu S, Hou L, Li Z, Li J, Xu J, Ren Z. Effect of Lactobacillus plantarum HT121 on serum lipid profile, gut microbiota, and liver transcriptome and metabolomics in a high-cholesterol diet-induced hypercholesterolemia rat model. Nutrition. 2020 Nov-Dec;79-80:110966.

\section{Figures}

A
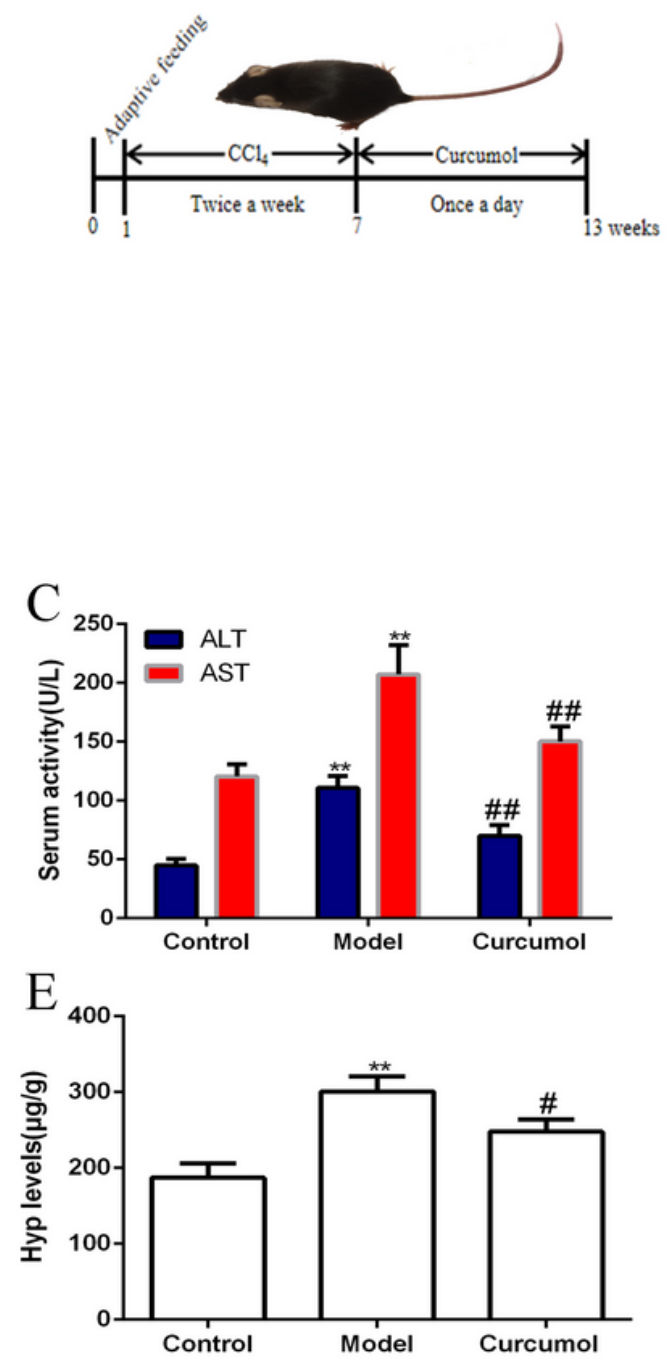
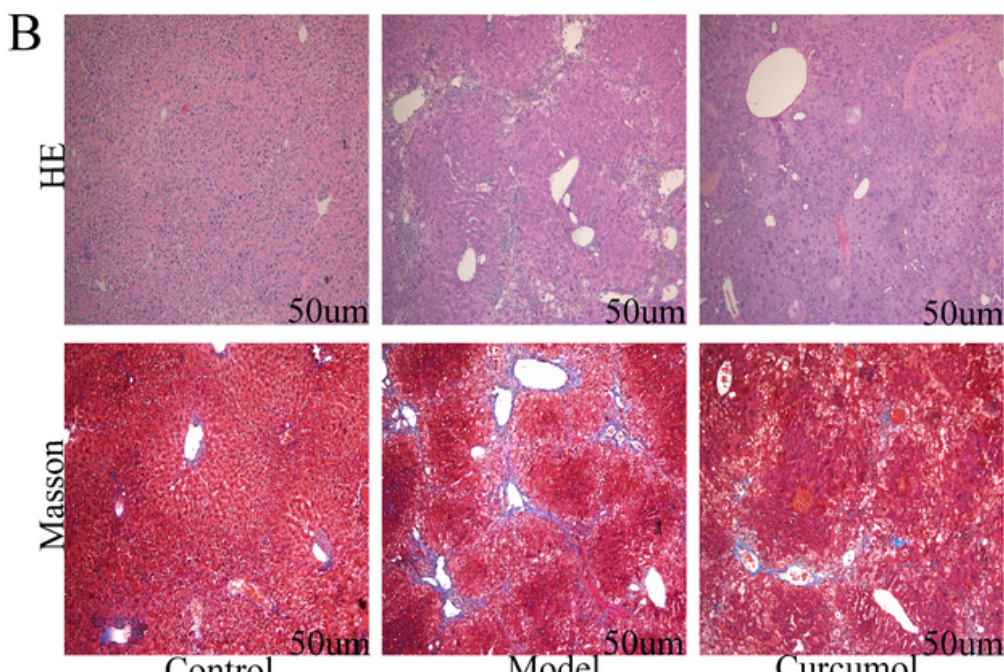

Control

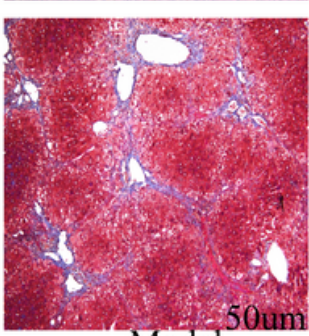

Model

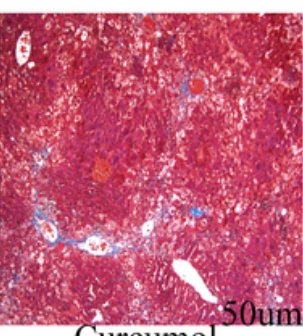

Curcumol
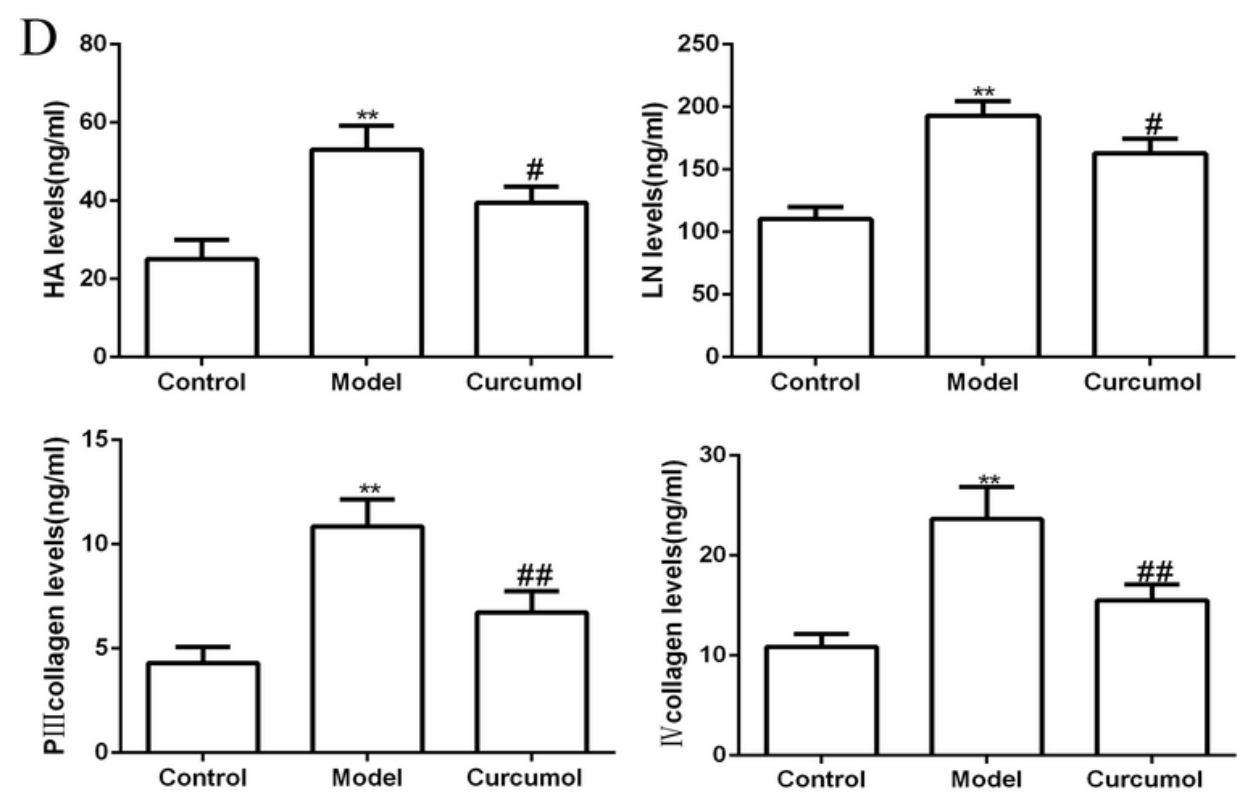

\section{Figure 1}

Effect of curcumol on pathological changes of liver tissue. A: Preparation of the liver fibrosis model and the drug intervention process. B: H\&E and Masson staining, Scale bar $=50 \mu \mathrm{m}$. C: Liver function via AST and ALT expression. D: Four indicators of liver fiber expression. E: Hydroxyproline expression. $\mathrm{n}=8,{ }^{\star} P<$ $0.05,{ }^{\star \star} P<0.01$ versus Control; ${ }^{\#} P<0.05,{ }^{\# \#} P<0.01$ versus Model. 

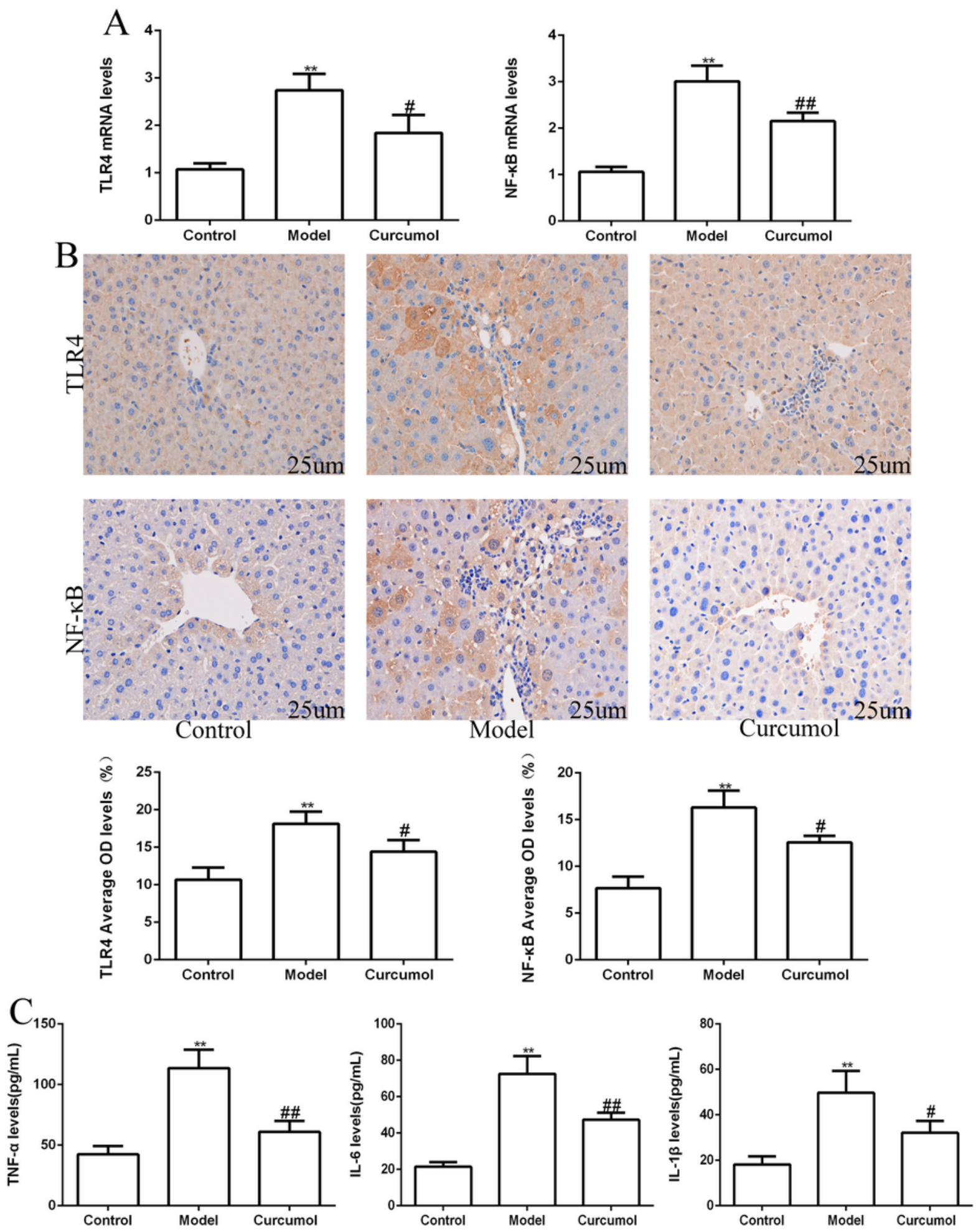

Figure 2

The effect of curcumol on the inflammatory signal and its downstream inflammatory factors. A: TLR4 and NF-KB mRNA expression. B: TLR4 and NF-KB protein expression. Scale bar $=25 \mu \mathrm{m}$. C: Expression levels of TNF-a, IL-6, and IL-1 $\beta . \mathrm{n}=8 .{ }^{*} P<0.05,{ }^{* \star} P<0.01$ versus Control. ${ }^{\#} P<0.05,{ }^{\#} P<0.01$ versus Model. 

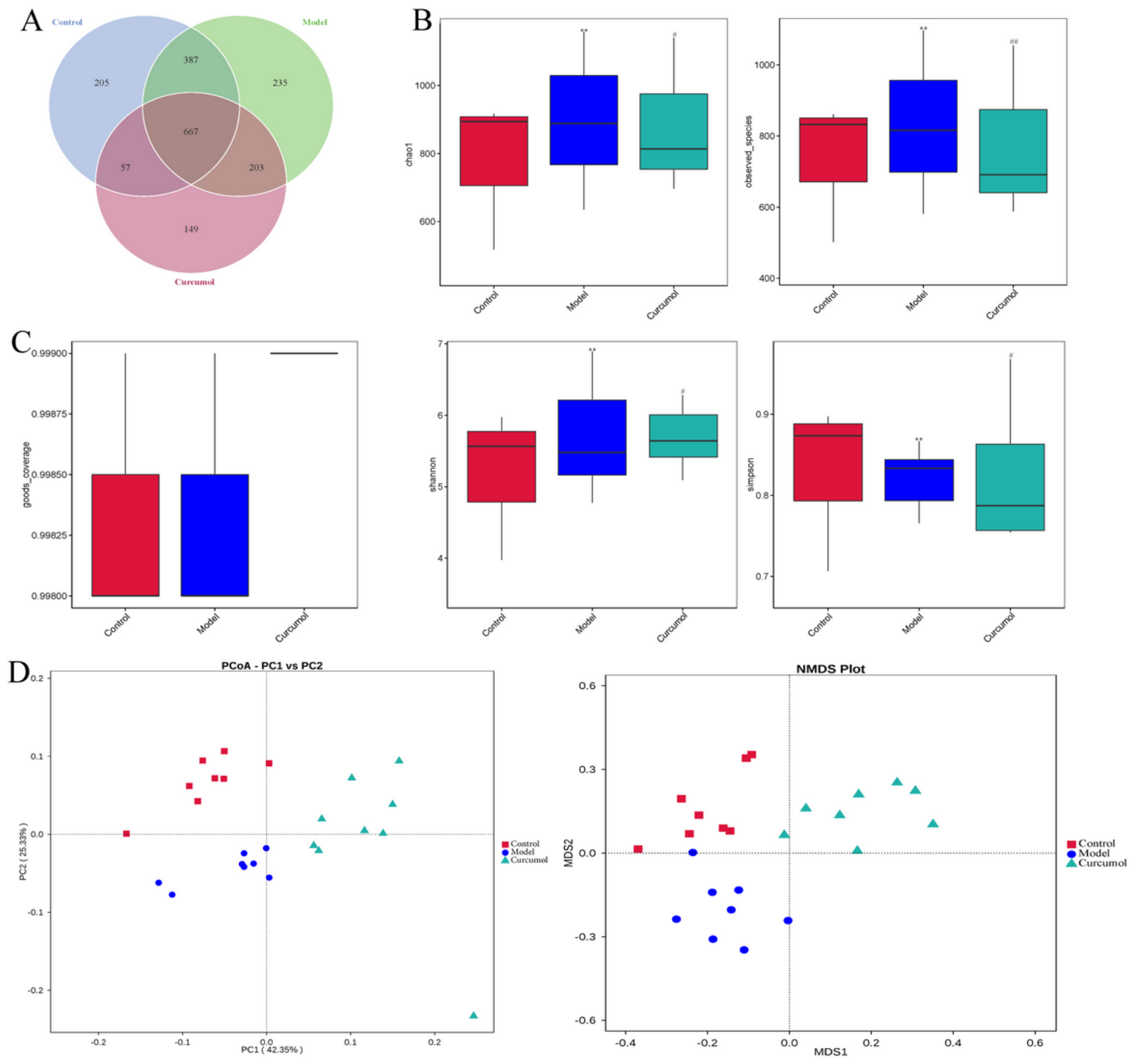

Figure 3

The effect of curcumol on the $a$ and $\beta$ diversity of intestinal flora. A: OTU expression in each group. B:

Chao1, Observed species, Shannon, and Simpson indexes. C: Sequencing depth of each group. D: PCoA and NMDS analysis of each group. $\mathrm{n}=8 .{ }^{*} P<0.05,{ }^{* \star} P<0.01$ versus Control. ${ }^{\#} P<0.05,{ }^{\# \#} P<0.01$ versus Model. 

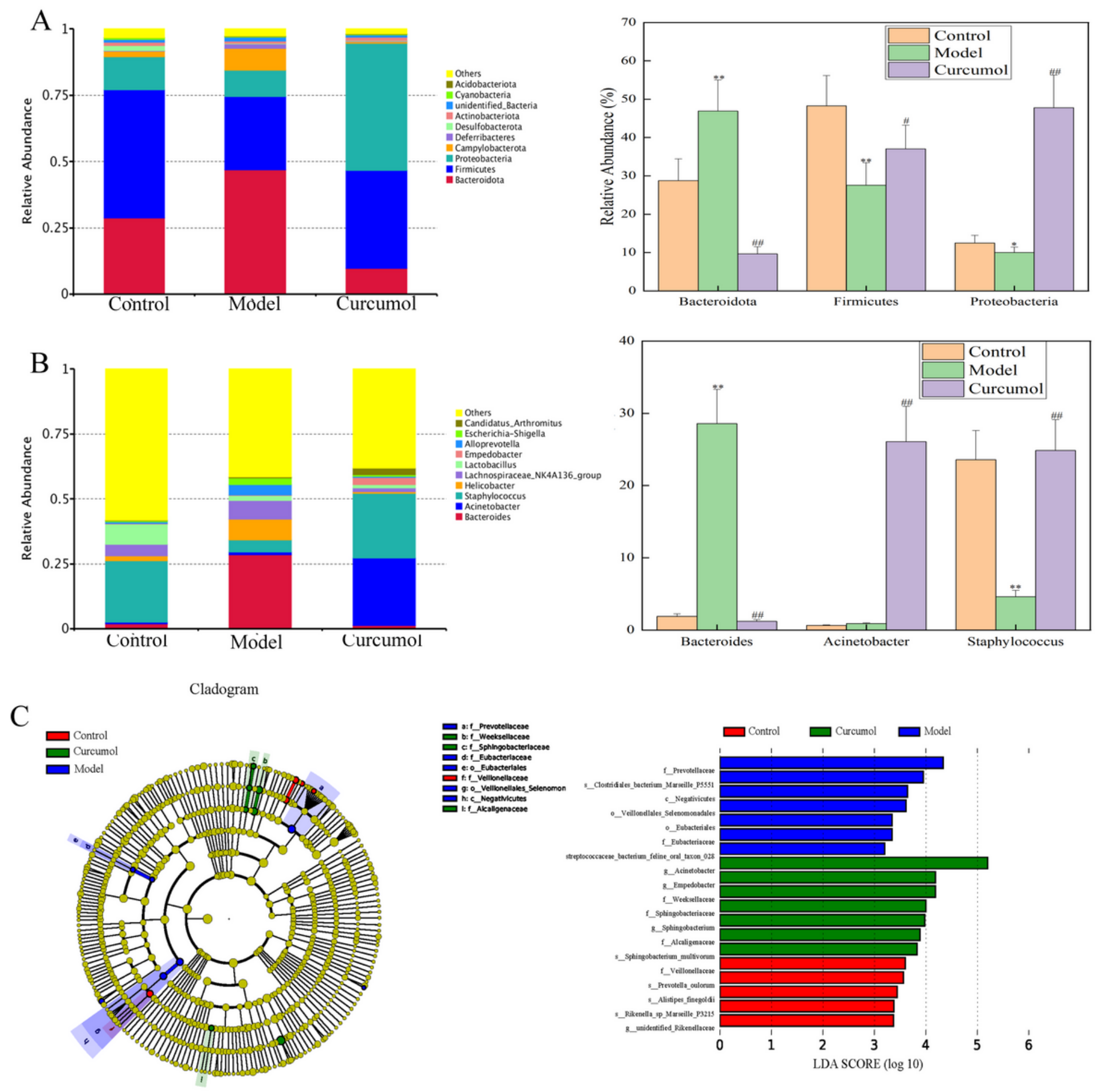

\section{Figure 4}

The effect of curcumol on intestinal flora abundance and specific taxa. A: Differences in the abundance of bacteria in each group at the phylum level (Top 10). B: Differences in the abundance of bacteria in each group at the genus level (Top 10). C: The expression of important flora in each group. $\mathrm{n}=8$. ${ }^{\star} P<$ $0.05,{ }^{* *} P<0.01$ versus Control. ${ }^{\#} P<0.05,{ }^{\# \#} P<0.01$ versus Model. 

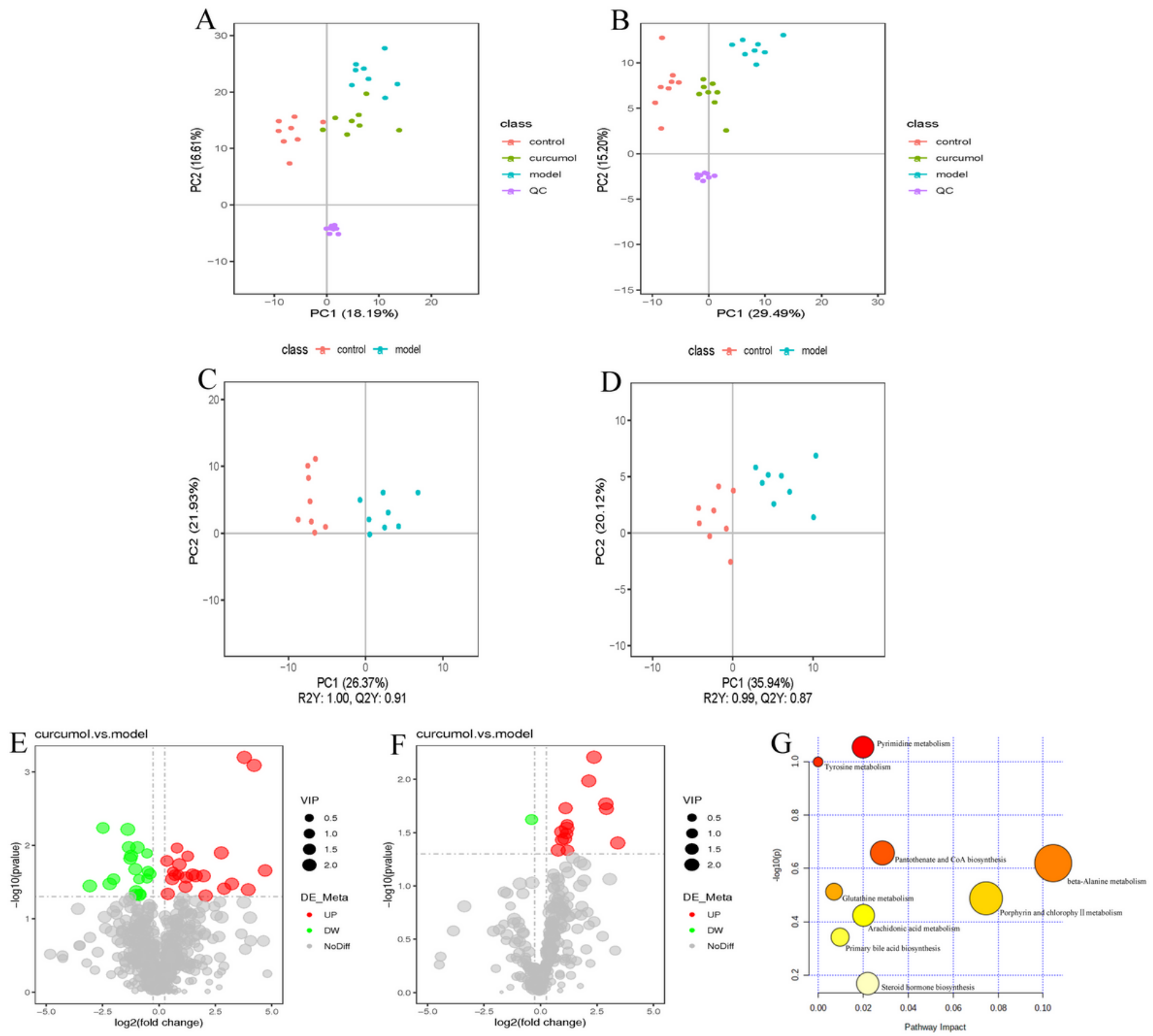

Figure 5

Effect of curcumol on metabolites of liver fibrosis in mice. A: PCA analysis of sample quality control in positive ion mode. B: PCA analysis of sample quality control in negative ion mode. C: PLS-DA analysis of differential metabolites between the model group and the blank group under positive ion mode. D: PLSDA analysis of differential metabolites between the model group and the blank group under negative ion mode. E: Volcano plot of the differential metabolites between the curcumol group and the model group under positive ion mode. F: Volcano plot of the differential metabolites between the curcumol group and the model group under negative ion mode. G: Enrichment analysis of differential metabolite pathways between the curcumol group and the model group. 

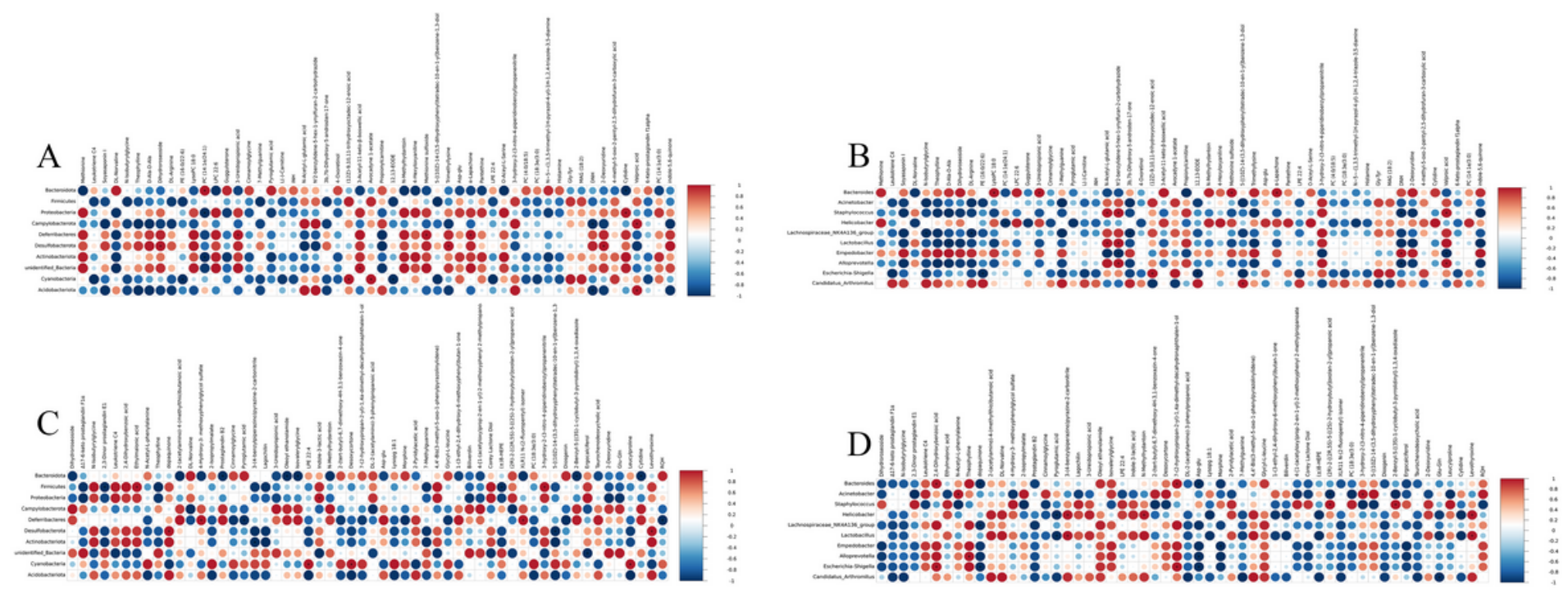

Figure 6

Association analysis of intestinal flora and metabolites. A: Correlation analysis of intestinal flora and differential metabolites between the model group and the blank group at the phylum level. B: Correlation analysis of intestinal flora and differential metabolites between the model group and the blank group at the genus level. C: Correlation analysis of intestinal flora and differential metabolites between the curcumol group and the model group at the phylum level. D: Correlation analysis of intestinal flora and differential metabolites between the curcumol group and the model group at the genus level. Blue indicates a negative correlation, and red indicates a positive correlation. The blank area in the figure indicates a P-value $>0.05$, and the area marked by an asterisk $\left(^{*}\right)$ indicates $P \leq 0.05$.
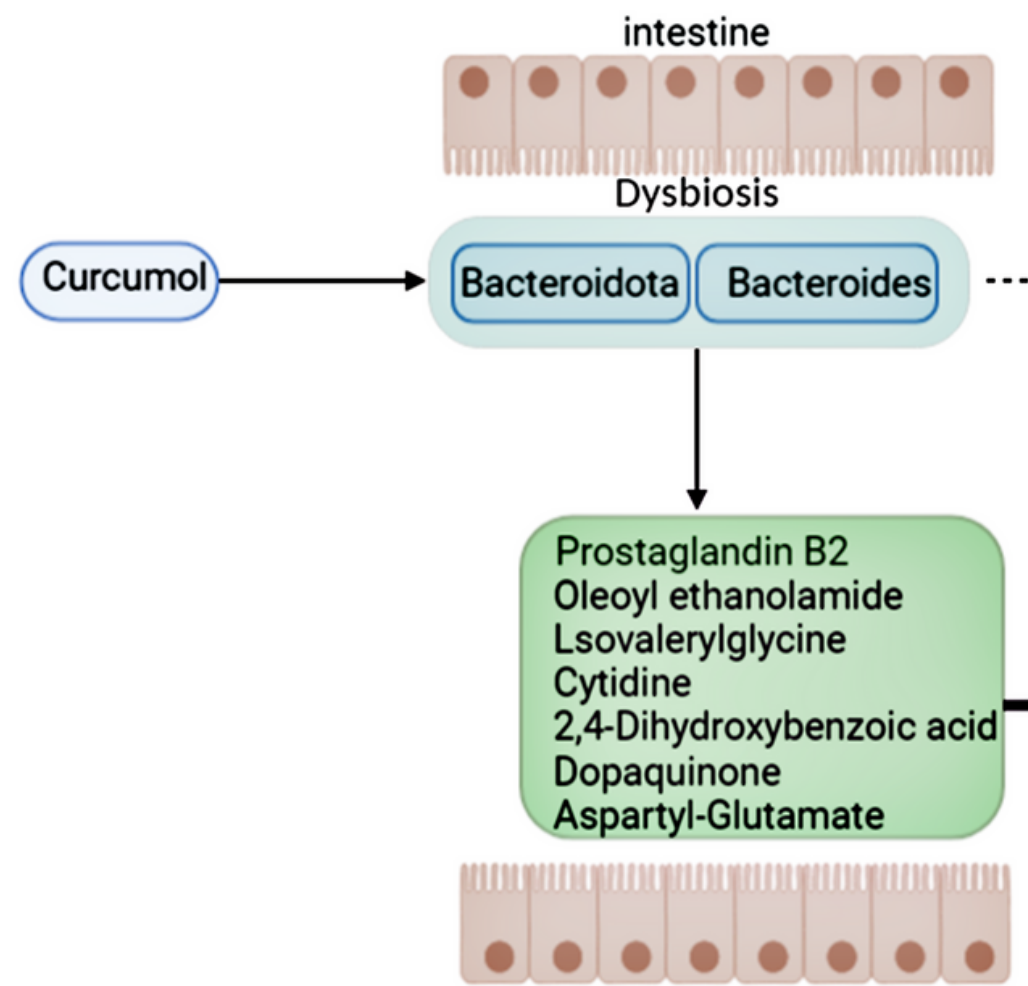
Figure 7

Curcumol regulates the intestinal flora and metabolic network to exert its anti-liver fibrosis mechanism.

\section{Supplementary Files}

This is a list of supplementary files associated with this preprint. Click to download.

- AuthorChecklistFull.pdf 